\title{
Impedance Spectroscopy Analysis using CNLS fitting for lithium bismuth borate glass containing 10 mol\% d-Block Nickel \& Vanadium Ions
}

\author{
Kanika $^{1}$, Manoj Duhan ${ }^{2}$, Satish Khasa ${ }^{3}$ \\ M.Tech Scholar, Electronics \& Communication Engineering Department, Deenbandhu Chhotu Ram University of \\ Science and Technology, Murthal, Sonepat ${ }^{1}$
}

Professor, Electronics \& Communication Engineering Department, Deenbandhu Chhotu Ram University of Science and Technology, Murthal, Sonepat ${ }^{2}$

Professor, Department of Physics, Deenbandhu Chhotu Ram University of Science and Technology, Murthal, Sonepat ${ }^{3}$

\begin{abstract}
This paper proposes a unique equivalent circuit model of Lithium Bismuth Borate Glass containing 10mol\% Nickel and Vanadium ions using LEVM engine by CNLS fitting. A lithium bismuth borate glass framework is processed by the conventional melt quenching method. In this paper considerable effort was put into the development of equivalent circuit model using Impedance Spectroscopy. The result of research revealed that a very good fit circuit is achieved with Immittance parameters.
\end{abstract}

Index Terms: Immittance Spectroscopy (IS), Lithium Bismuth Borate Glass (LBBG), Complex Nonlinear Least Square Fitting (CNLS), Distributed Elements (DEs).

\section{INTRODUCTION}

Impedance Spectroscopy deals with the complex quantities. IS not only deals with the impedance but four other functions are summed together, thus IS is also called as Immittance Spectroscopy. These four functions are Impedance, Admittance, complex dielectric constant and complex modulus. IS includes the measurement of small signal frequency response of electrolytes, semiconductors, dielectrics and biological cells [1]. In this paper Impedance Spectroscopy is expressed in two forms: Rectangular and Modulus. IS is classified in two categories: Dielectric and Electrochemical Impedance Spectroscopy. These are used in chemical, biochemical and medical research field for analyzing organic compounds [4].

In this paper we use Lithium Bismuth Borate Glass containing 10mol\% Nickel and Vanadium ions for Impedance Spectroscopy Analysis using CNLS fitting. This glass is developed through melt- quench way[5]. Borate is one of the most important glass and has been contained in many types of glass systems to attain chemical and physical properties. Different dielectric parameters like electrical modulus $(\mathrm{M})$, loss tangent $(\tan \delta)$, dielectric losses (E) etc are calculated and their fluctuation with temperature and frequency are analyzed. In this work, an attempt has been made for the preparation and characterization of bismuth-borate glasses with the addition of lithium oxide as modifier.

Complex Nonlinear Least Square (CNLS) fitting fits complex data to a model. This is the new graphical method for analyzing impedance data presented in the complex plane plot[3]. The aim of least squares fitting procedures is to find a set of parameters $\mathrm{P}$ which will minimize the sum:

$$
\mathrm{S}(\mathrm{P})=w_{j}[\mathrm{Yj}-\mathrm{YCj}(\mathrm{P})]^{2}
$$

where the sum is taken over $1 \ldots . . \mathrm{M}$, where $\mathrm{M}$ is the total number of data points, $\mathrm{wj}$ is the weight associated with the jth point, $\mathrm{Yj}$ is the jth data point value to be fitted, and $\mathrm{YCj}(\mathrm{P})$ is the corresponding value of the calculated fitting function The advantage of our approach is that it can be employed easily with commonly available spreadsheet calculation software like Microsoft Excel[2]. The most important advantage of the CNLS is the possibility to fit the entire impedance function in the whole range of frequencies to a model the equivalent circuit. Steps for CNLS fitting /simulation:

1. FIT: CNLS fitting fits real part, imaginary part and frequency response data of appropriate model.

2. GET: Objective parameter estimates, their estimated standard deviation (SD), correlation and SD of fit.

3. NO: Subjective graphical, extrapolative and subtractive procedures needed. 
4. AFTER: fitting, one can analytically separate out the various contributions to the system: electrode effects, Conductive System dispersion data (CSD) response, Dielectric System Dispersion Data (DSD) response and DC conductivity.

\section{LEVM/LEVMW TOOL SUMMARY}

LEVM is not a acronym but a name. LEVMW is a new window version of LEVM. The early history of LEVM program begins with the paper by Macdonald and Garber, J. Electrochem. Soc.124 (1977). The first version of LEVM were called LEVM-OLSON or LOMFP, but in 1988 the OLSON part of the fitting program was eliminated and a much superior nonlinear least-squares iteration procedure was introduced. The present version is 8.13 was updated in May, 2015. LEVM is powerful measuring tool for CNLS fitting of Impedance Spectroscopic data. LEVM is a computer program for complex non linear -least squares data fitting, simulation, analysis of high resistivity - disordered materials, glasses, polymers and dielectrics, fits complex, real and imaginary frequency response data. LEVMW includes all the original LEVM program.

LEVM program includes mainly four functions. First, it may be used to estimate distribution of relaxation time (DRT) or activation energies (DAE) from frequency or transient response data. It may also be used for simulation of circuits and other model response functions and it may be used to fit frequency response or transient response data by complex nonlinear least squares(CNLS) or nonlinear squares(NLS). All the output results may be plotted in 2-D or 3-D form at any immittance level[2]. LEVM unified larger number of choices, contributing greatly to flexibility and power. It can easily select the fitting models.

LEVM works directly with any Windows operating system from W-95 TO W-8. LEVM program runs on a PC or MS-DOS using the Microsoft FORTRAN PowerStation V.1 compiler and also compiled on V.40 but the results only run on above mentioned Windows. The present version of LEVM handles data with a maximum of 2002 data points (1001 real and 1001 imaginary components) and upto 42 free parameters. It requires extended memory. A smaller version of LEVM involves a maximum of 150 points and 28 free parameters and operate with ordinary memory. LEVM provides 37 distributed complex circuit elements (DCEs or DEs).It is also used to fit data to an appropriate response model which contains several temperature dependent parameters[2]. LEVMW involves LEVMWIN.EXE, a powerful fitting engine and front end graphic interface programs, LEVMRUN.EXE., a powerful plotting program for LEVMW outputs and residuals, and LEVMVIEW.EXE, as well as LEVM.HLP.

\section{A. CHARACTERISTICS OF LEVM/LEVMW}

Ability to determine parameters of the error variance model, i.e. weighting parameters.

Greater than 10 weighting choices are available.

Estimation of distributions is possible by powerful methods of inversion of frequency or transient response data. Input/Output may be in rectangular, polar or log-polar form.

Greater than 30 different distributed circuit elements(DCEs) are available for different circuits. It estimates the parameters of a distribution of relaxation times or activation energies.

Graphical presentation of spectra, results and parameters is also included.

MEISP (Multiple EIS parameterization) software package also uses LEVM engine for fitting.

The fitting and simulation may be carried at immittance level.

It is also applicable for dielectric, conductive and mixed systems.

\section{B. LEVMW INITIALIZATION STEPS}

It uses a specific type of input file for performing CNLS fitting of given data.

The input file contains two parts i.e. top part listing the fitting choices and bottom part contains three data columns: frequency, real part, imaginary part (or frequency, magnitude and phase).

The input file assigned which one of the available circuits is suitable for fitting and simulation.

After selecting fitting circuit initial values must be entered for those free parameters used for fit.

Data are input in LEVM in the form of impedance, admittance, modulus and dielectric constant or complex capacitance.

\section{INPUT FILE}

MKLIO combines the top part of LEVM input file with arbitrary data file to produce a new complete input file. A complete input file includes a header and below that a data section. The header part requires that a number of choices be made and estimated values of free and fixed parameters. Two main points while preparing input file are: a) You must choose appropriate fitting model from possible circuits and b) Data must be combined with LEVM input header file to make fully executable input file. 
UGC Approved Journal

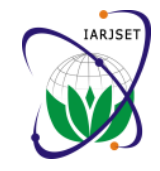

IARJSET

ISSN (Online) 2393-8021

ISSN (Print) 2394-1588

International Advanced Research Journal in Science, Engineering and Technology

ISO 3297:2007 Certified

Vol. 4, Issue 6, June 2017

\section{GRAPHICS INFORMATION}

A very useful addition to LEVM is the 2-D and 3-D plotting program. Its results are coloured and carries all necessary data transformation and even rotate 3-D plots if required [2]. A new 2-D option has been added to allow the plotting of the absolute value of the tangent of the data (only in rectangular form) (OTN) or its inverse (ITN). CPTRAN(FORTRAN program is used to plot the data and represent the results with the help of graphs.

\section{E. OUTPUT FILES}

LEVM produces screen output during operation this is incomplete and the main output appears in the files are AUXPNTL and PNTOUTL. After observing above files the two standard deviations, SF: PRSDAV (weighting residuals) and PRSDRMS (relative residuals) are automatically induced at the end of the third line of OUTIN and if they exceed 0.2 or 0.3 , it is a poor fit. For a good fit, one would expect 0.03 or less. A fit quality factor (FQF) value is also given to help in comparing two fits[2].

AUXPNTL is for auxillary output and allows user to convert data between 16 different modes. It is a secondary output file which contains list of parameter inputs and includes a list of the data, the fitted results, and the fitting residuals of each point[2].

PNTOUTL is used for main output printing. The PNTOUTL file is obtained by running AZC. You will need to first find the circuit model used (here B) and then use the identification list for this circuit in order to pair the parameter values with the actual elements in the B circuit used in the fitting (i.e., all non-zero parameters in the PNTOUTL initial listing). Only parameter values with a 1 or 2 after them are free during the fitting. The more free parameters in a fit more desirable the fit that their initial value is close to their final converged values. LEVM can converge when parameter estimates are far from their correct values, the more free parameters in the fitting, the more difficult it is for the program to converge to the correct set of parameter estimates.

\section{PROPOSED WORK}

There are five major steps in the proposed work of our research:
A. Data Collection
B. Input File Preparation
C. Pre- Analysis
D. Resetting of parameters
E. Equivalent Circuit Model

A. Data Collection

The first step of our analysis is to collect the datasheet of Lithium Bismuth Borate Glass parameters. There are many distributed elements available designed to be use in Immittance Spectroscopy.

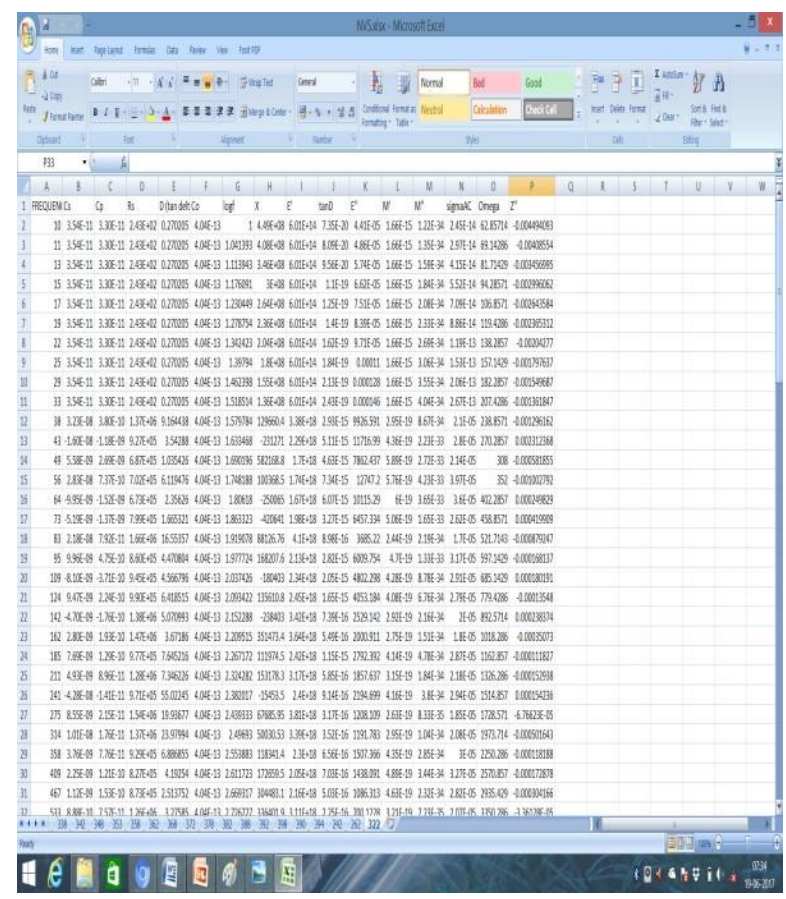

Fig.1 Datasheet of LBBG 


\section{UGC Approved Journal}

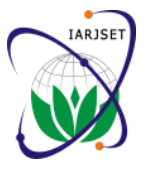

These discrete elements are resistance, capacitance, inductance, modulus, relaxation time constant, dielectric constant etc but here mainly we use Resistance and Capacitance parameters for our work. These parameters may be free or fixed fitting parameters. The impedance spectroscopy of our dielectric glass is carried out at temperature of $322^{\circ} \mathrm{C}$ and frequency range of $1 \mathrm{MHz}-5 \mathrm{MHz}$.This datasheet was provided by Physics Department which is shown in fig.1.

\section{B. Input File Preparation}

To fit our own data to a model we have to choose appropriate one, prepare the top part of an input file and combine it with our bottom part of file which contains our data to make the final LEVM input file. Our data should be arranged in three columns: frequency, real part, imaginary part and if the imaginary part of the data is positive, it needs to be made negative in the final output file as shown in fig.1. In input file preparation we need to decide which model parameters should be used and which should be free or fixed.

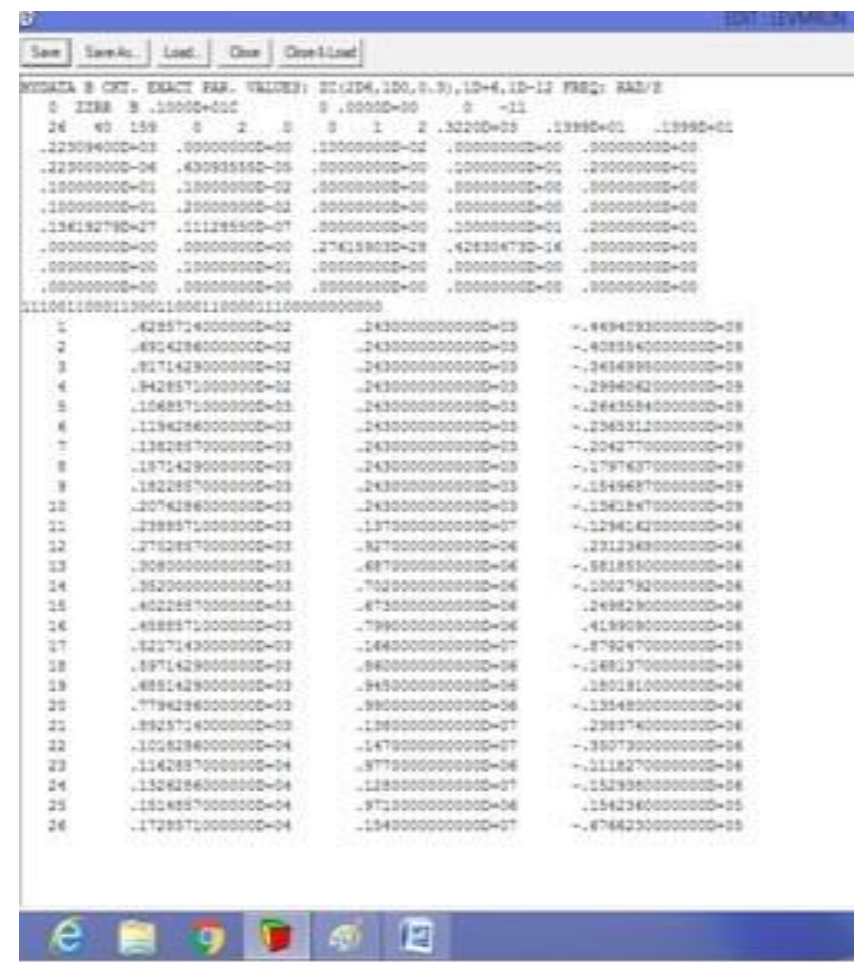

Fig. 2 Prepared Input File for $\mathrm{LBBG}$ at $322^{\circ} \mathrm{C}$

C. Pre Analysis

At this level, the output obtained as a result of the initial guesses of the parameters values in the top part of the input file is determined. The quantities, PRSDAV and PRSDRMS are expected to be 0.03 or less for a good fit otherwise it is not a good fit.

\section{Resetting of Parameters}

Values of standard deviations are automatically induced at the end of OUTIN line. If these values be less than or equal to 0.03 than no need to change the parameters values otherwise we need to change the values of fixed or free parameters in ADVANCED EDITOR section.

\section{E. Equivalent Circuit Determination}

After performing above steps we have to select the best fit circuit from circuits A,B,C upto T. Here we determine the circuit $\mathrm{B}$ equivalent circuit because values of PDAV and PDRMS is less than 0.03.In the equivalent circuit DE represents the distributed/discrete elements which may be in series or parallel combination.

\section{RESULTS}

Besides screen output, ordinary frequency response produces several output files. The most important is shown in fig. 3 i.e. PNTOUTL file which shows the values of two standard deviations less than 0.03 these values are $2.8786 \mathrm{D}$ 02 and 1.8838D-02 which shows that circuit we select is good fit and equivalent circuit of this model is shown in fig.6. 
UGC Approved Journal

ISO 3297:2007 Certified

Vol. 4, Issue 6, June 2017
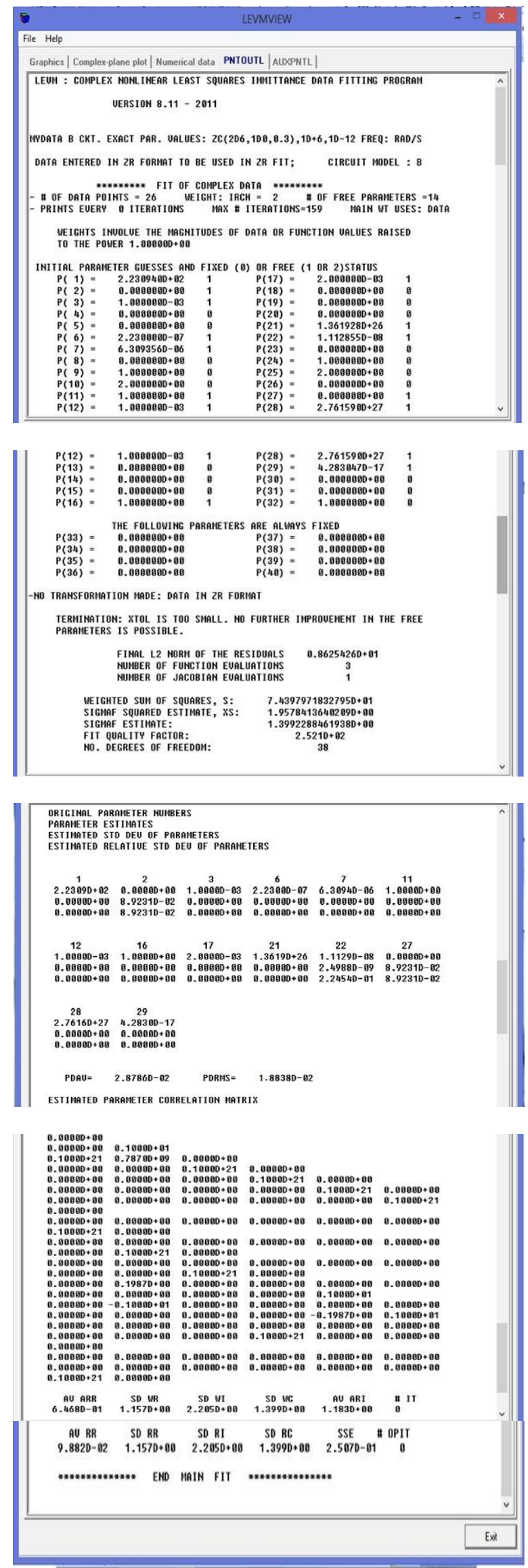

Fig.3 PNTOUTL File obtained for the fit 
UGC Approved Journal

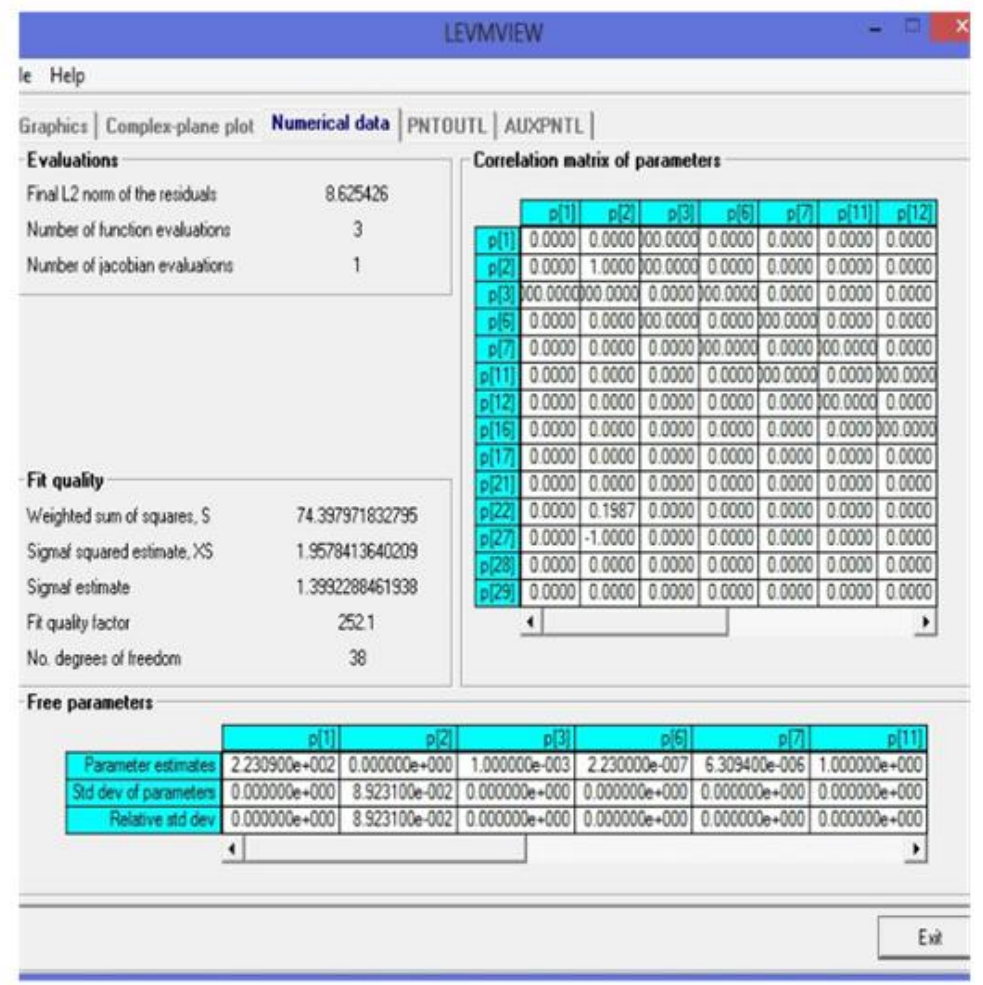

Fig.4 Numerical data output for the fit performed

Fig 4 shows the correlation matrix of parameters and the free parameters variations standard deviations with Fit Quality Factor (FQF)
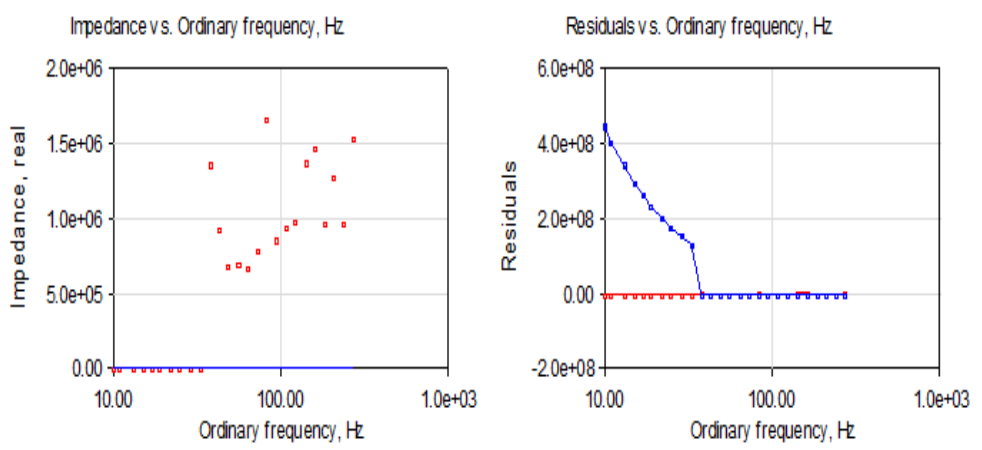

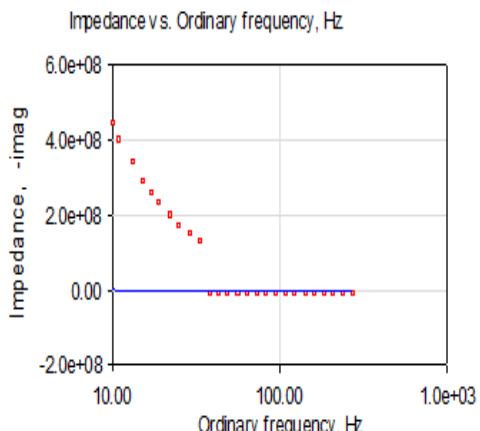

(a)

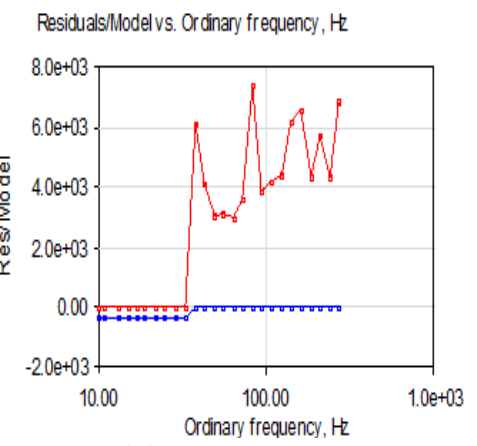

(b)

Fig.5 a) Impedance v/s frequency curve of $\mathrm{LBBG}$ at $322^{\circ} \mathrm{C}$; b) Residuals v/s frequency curve for $\mathrm{LBBG}$ at $322^{\circ} \mathrm{C}$

Fig. 5 shows Direct graphical output obtained from LEVMW CNLS fitting of the material used at temp. $322^{\circ} \mathrm{C}$ The left two plots show the input data as dots and fitting result as solid lines. The right two plots show the real part residuals and relative residuals by red lines and imaginary part by blue lines. 
UGC Approved Journal

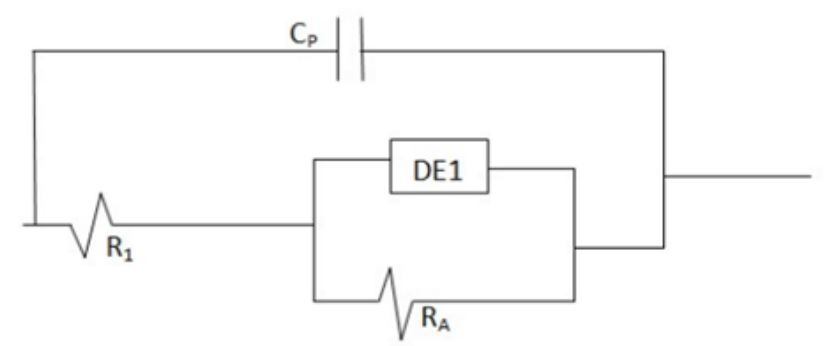

Fig.6 Proposed Equivalent Circuit Model of LBBG at $322^{\circ} \mathrm{C}$

Fig.6 shows the equivalent circuit model of LBBG at temperature of $322^{\circ} \mathrm{C}$. Here, is only one distributed element is connected in series with resistance and in parallel with capacitance. These DEs are RDE, TDE, NDE, PDE, UDE and there corresponding values are $0.88067826 \mathrm{D}+04 \Omega, 0.43469930 \mathrm{D}+06,0.20000000 \mathrm{D}+01,0.11916233 \mathrm{D}+02$ and $0.00000000 \mathrm{D}+00, \mathrm{R} 1$ is $0.80760714 \mathrm{D}+03, \mathrm{RA}$ is $0.13792097 \mathrm{D}-03$, CP is $0.89393093 \mathrm{D}-16$ and NELEM is 2 (so that the DE be simply a combination of resistor and capacitor in parallel). The changes allows us to better circuit elements.

\section{CONCLUSION}

This paper deals with the designing of equivalent circuit model for Lithium Bismuth Borate Glass based on the impedance spectroscopic data, the circuit model, fitting circuit choices made in the input file and the parameter values assigned in the input file. A new accurate method of determining equivalent circuit of dielectric material using CNLS fitting is LEVM/LEVMW. The circuit is very simpler using a convention: Any element with a parameter value set to zero is assumed to be not present.

\section{FUTURE SCOPE}

Future scope of this research work is to measure the larger signal frequency response of semiconductors, electrolytes. We should make the measurement of the same material at same temperature using two or more electrodes. Other quantities like light, magnetic field can be used for further analysis. In future we add further weighting choices to least squares to minimize the sum of the absolute values of the residuals.

\section{REFERENCES}

[1] "Impedance Spectroscopy," in 1991 Yearbook of Encyclopedia of Physical Science and Technology, R.A. Meyers, Editor, Academic Press, San Diego, CA(1991),pp.279-291.Republished with additions in Ann. of Biomed. Eng.20, 289- 305(1992), March 20, 2017, 10am.

[2] http://jrossmacdonald.com/jrm/wp-content/upl- oads /LEVMMANUAL-5-12-14.pdf, January 25, 2017, 5pm.

[3] Macdonald, Schoonman, and Lehnen, Solid State Ionics 5 (1981) 137; J. Electroanal. Chem. 131(1982)77.

[4] https://www.scribd.com/document/235057508/1 77-Impedance-Spectroscopy, June 19, 2017, $1 \mathrm{pm}$.

[5] http://file.scirp.org/pdf/NJGC_20160405171113 13.pdf, June 20, 2017, 2am. 